\title{
Polinômios ortogonais e fórmulas de quadratura no círculo unitário e no intervalo $[-1,1]$
}

\author{
Charles F. dos Santos ${ }^{*} \quad$ Vanessa Paschoa \\ Departamento de Ciência e Tecnologia, ICT, UNIFESP, \\ 12231-280, São José dos Campos, SP \\ E-mail: charles.ferreira@unifesp.br, vanessa.paschoa@unifesp.br.
}

\section{RESUMO}

Neste trabalho apresentaremos como obter as fórmulas de quadratura de Gauss (com extensão aos casos de Radau e Lobatto) a partir de fórmulas de quadratura no círculo unitário.

Polinômios ortogonais no círculo unitário Denote por $\mathbb{T}=\{z \in \mathbb{C}:|z|=1\}$ o círculo unitário do plano complexo. Seja $\omega:[-\pi, \pi] \rightarrow \mathbb{C}$ uma função tal que a forma $\langle\rangle:, \mathbb{P}(\mathbb{C}) \times \mathbb{P}(\mathbb{C}) \rightarrow \mathbb{C}$ dada por

$$
\langle p, q\rangle=\int_{-\pi}^{\pi} p\left(e^{i \theta}\right) \overline{q\left(e^{i \theta}\right)} \omega(\theta) \mathrm{d} \theta
$$

defina um produto interno no espaço vetorial $\mathbb{P}(\mathbb{C})$ das funções polinomiais complexas. Diremos que $\omega$ é uma função peso em $\mathbb{T}$. Uma sequência de polinômios $\left(\Phi_{n}(z)\right)_{n \geq 0}$ tal que $\Phi_{n}(z)$ tem sempre grau $n$, $\left\langle\Phi_{n}, \Phi_{k}\right\rangle=0$ se $n \neq k$ e $\left\langle\Phi_{n}, \Phi_{n}\right\rangle$ é real positivo é chamada sequência de polinômios ortogonais em relação a $\omega$. A exigência de que todos os $\Phi_{n}(z)$ sejam mônicos garante a unicidade da sequência.

As propriedades elementares dos $\Phi_{n}$ são tratadas, por exemplo, em [3]. Entre elas, está o fato de que os zeros de $\Phi_{n}(z)(n \geq 1)$ pertencem ao disco unitário aberto, ou seja, têm módulo menor que 1 . Merecem destaque também as fórmulas de recorrência, dadas por

$$
\begin{gathered}
\Phi_{n+1}(z)=z \Phi_{n}(z)+\Phi_{n+1}(0) \Phi_{n}^{*}(z), \\
\Phi_{n+1}(z)=\left(1-\left|\Phi_{n+1}(0)\right|^{2}\right) z \Phi_{n}(z)+\Phi_{n+1}(0) \Phi_{n+1}^{*}(z),
\end{gathered}
$$

para $n \geq 1$, assumindo aqui que todos os $\Phi_{n}$ são mônicos. A notação * se refere ao polinômio recíproco, definido por $p^{*}(z)=z^{n} \bar{p}\left(z^{-1}\right)$, onde $n$ é o grau do polinômio $p(z)$.

Quadratura de Szegó Considere agora o problema de aproximar uma integral em $\mathbb{T}$ por uma fórmula que dependa apenas dos valores do integrando em pontos pré-fixados, ou seja,

$$
\int_{-\pi}^{\pi} f\left(e^{i \theta}\right) \omega(\theta) \mathrm{d} \theta \approx \sum_{k=0}^{n} \lambda_{k} f\left(z_{k}\right),
$$

onde $\omega$ é uma função peso em $\mathbb{T}$, os $\lambda_{k}$, chamados pesos, não dependem de $f$ e os $x_{k}$, chamados nós, também são fixos, $k=1, \ldots, n$. Uma fórmula do tipo acima é chamada fórmula de quadratura, e se houver "=" no lugar de " $\approx$ " para um certa função $f$, dizemos que a quadratura é exata para $f$.

É possível construir fórmulas de quadratura de $n$ nós que sejam exatas para todas as funções em um certo espaço vetorial de dimensão $2 n-1$; além disso, tal espaço é maximal. No entanto, diferentemente do que ocorre com polinômios ortogonais na reta, não se pode usar como nós os zeros dos polinômios ortogonais com relação a $\omega$, uma vez que eles não pertencem a $\mathbb{T}$.

Dado $n \geq 1$, considere o polinômio $\Phi_{n}(z)+\tau \Phi_{n}^{*}(z)$, onde $|\tau|=1$. Seus zeros são sempre distintos e pertencem a $\mathbb{T}$, portanto podem ser usados como nós da quadratura.

*bolsista de Iniciação Científica FAPESP 
Teorema. Denote $\Lambda_{-(n-1), n-1}=\operatorname{span}\left\{z^{k}:-(n-1) \leq k \leq n-1\right\}$. Seja $\omega$ uma função peso em $\mathbb{T} e$ fixe $n \geq 1$ e $\tau \in \mathbb{T}$ qualquer; sejam $\xi_{1}, \ldots, \xi_{n}$ as raízes de $\Phi_{n}(z)+\tau \Phi_{n}^{*}(z)$. Existem $\lambda_{1}, \ldots, \lambda_{n}$ fixos, que são reais positivos, tais que

$$
\int_{-\pi}^{\pi} f\left(e^{i \theta}\right) \omega(\theta) d \theta=\sum_{k=1}^{n} \lambda_{k} f\left(\xi_{k}\right):=S_{n}(f), \quad \forall f \in \Lambda_{-(n-1), n-1} .
$$

Por outro lado, se uma fórmula de quadratura com n nós é exata para toda $f \in \Lambda_{-(n-1), n-1}$, então seus nós são as raízes de $\Phi_{n}(z)+\tau \Phi_{n}^{*}(z)$, onde $|\tau|=1$.

Por fim, não existe fórmula de quadratura exata para toda $f \in \Lambda_{-(n-1), n}$ ou toda $f \in \Lambda_{-n, n-1}$.

A regra de quadratura $S_{n}$ como no teorema acima se chama fórmula de quadratura de Szegó.

Obtendo quadraturas em $\mathbb{T}$ a partir da quadratura em $[-1,1]$ Seja $\sigma$ uma função peso no intervalo $[-1,1]$. Definindo $\omega(\theta)=\sigma(\cos \theta)|\sin \theta|$, temos que $\omega$ é uma função peso no círculo unitário, é simétrica e seus polinômios ortogonais mônicos $\Phi_{n}(z)$ têm coeficientes todos reais. É possível obter os polinômios ortogonais em relação a $\sigma$ a partir dos $\Phi_{n}$ ([3], teorema 11.5).

Considere as quadraturas de Gauss, Gauss-Radau e Gauss-Lobatto em $[-1,1]$, que têm a forma geral

$$
\int_{-1}^{1} F(x) \sigma(x) \mathrm{d} x \approx A f(1)+B f(-1)+\sum_{k=1}^{m} A_{k} f\left(x_{k}\right),
$$

onde $x_{k}=\cos \theta_{k}, k=1, \ldots, m$; no caso Gauss $A=B=0$ e $x_{k}$ são os zeros do $m$-ésimo polinômio ortogonal com relação a $\sigma(x)$, no caso de Gauss-Radau $A=0$ ou $B=0$ e $x_{k}$ são os zeros do $m$-ésimo polinômio ortogonal com relação a $(1-x) \sigma(x)$ ou $(1+x) \sigma(x)$ e no caso de Gauss-Lobatto $x_{k}$ são os zeros do $m$-ésimo polinômio ortogonal com relação a $\left(1-x^{2}\right) \sigma(x)$.

Teorema. Sejam $\sigma$ uma função peso no intervalo $[-1,1]$ e $\omega$ uma função peso no círculo unitário tais que $\omega(\theta)=\sigma(\cos \theta)|\sin \theta|, \theta \in(-\pi, \pi]$. Seja $S_{n}$ a fórmula de quadratura de Szegó para $\omega$ como em (1) $\operatorname{com} \xi_{k}=e^{i \theta_{k}}$.

(i) Se $n=2 m$ e $\tau=1$ então $S_{n}(f)=\sum_{k=1}^{m} A_{k}\left[f\left(\xi_{k}\right)+f\left(\overline{\xi_{k}}\right)\right]$ com $A_{k}$ e $\theta_{k}$ dados por (2) no caso Gauss.

(ii) Se $n=2 m$ e $\tau=-1$ então $S_{n}(f)=2 A f(1)+2 B f(-1)+\sum_{k=1}^{m-1} A_{k}\left[f\left(\xi_{k}\right)+f\left(\overline{\xi_{k}}\right)\right]$ com $A, B, A_{k}$ e $\theta_{k}$ dados por (2) no caso Gauss-Lobatto;

(iii) Se $n=2 m+1$ e $\tau=1$ então $S_{n}(f)=2 B f(-1)+\sum_{k=1}^{m} A_{k}\left[f\left(\xi_{k}\right)+f\left(\overline{\xi_{k}}\right)\right] \operatorname{com} B, A_{k}, \theta_{k}$ dados por (2) no caso Gauss-Radau;

(iv) Se $n=2 m+1$ e $\tau=-1$ então $S_{n}(f)=2 A f(1)+\sum_{k=1}^{m} A_{k}\left[f\left(\xi_{k}\right)+f\left(\overline{\xi_{k}}\right)\right] \operatorname{com} A, A_{k} e \theta_{k}$ dados por (2) no caso Gauss-Radau.

Pelo teorema acima, os pesos das fórmulas "tipo Gauss" coincidem com os das fórmulas de Szegő (a não ser por um fator 2 nos extremos) e seus nós são as partes reais dos nós da quadratura de Szegő.

Palavras-chave: Polinômios Ortogonais, Polinômios de Szegó, Quadratura Gaussiana, Quadratura de Szegö, Polinômios Para-Ortogonais

\section{Referências}

[1] A. Bultheel; L. Daruis; P. González-Vera, A connection between quadrature formulas on the unit circle and the interval $[-1,1]$, Journal of Comp. and Appl. Math., 132 (2001) 1-14.

[2] F. A. Martins, "Polinômios Para-Ortogonais e Análise de Frequência", Dissertação de Mestrado, IBILCE-Unesp, 2005.

[3] G. Szegô, "Orthogonal Polynomials”, AMS Colloquium Series, vol. 23, 4th ed., American Mathematical Society, Providence, RI, 1975. 